University of Wollongong

Research Online

Faculty of Engineering - Papers (Archive)

Faculty of Engineering and Information

Sciences

$1-1-2007$

\title{
Marital infidelity and its effect on pathogen diversity
}

Matthew J. Berryman

University of Wollongong, mberryma@uow.edu.au

Follow this and additional works at: https://ro.uow.edu.au/engpapers

Part of the Engineering Commons

https://ro.uow.edu.au/engpapers/4948

\section{Recommended Citation}

Berryman, Matthew J.: Marital infidelity and its effect on pathogen diversity 2007.

https://ro.uow.edu.au/engpapers/4948

Research Online is the open access institutional repository for the University of Wollongong. For further information contact the UOW Library: research-pubs@uow.edu.au 


\title{
Marital infidelity and its effect on pathogen diversity
}

\author{
Matthew J. Berryman \\ Centre for Biomedical Engineering and \\ School of Electrical and Electronic Engineering, \\ The University of Adelaide, SA 5005, Australia.
}

\begin{abstract}
Marital infidelity is usually examined solely in terms of strategies of men and women, with an emphasis on the enhanced payoff for male infidelity (provided he can get away with it). What are not clear are the strategies used, in terms of how often to engage in extra-marital affairs. It has been proposed that female strategies are governed by a "decision" to maximize the genetic diversity of her offspring, in order to better guarantee that at least some will survive against a common pathogen. This strategy would then impact on the strategies and diversity of pathogens. I make a number of predictions about both strategies and the genetic diversity of humans and pathogens, couched in game-theoretic terms. These predictions are then compared with the existing evidence on the strategies used by women and also in terms of the genetic diversity of human populations.
\end{abstract}

Keywords: infidelity, co-evolution, adaptation, pathogens, diversity

\section{INTRODUCTION}

There is a wealth of evidence from the natural world that environmental influences, including pathogens, can affect mating strategies in both plants and animals. ${ }^{1-3}$ In particular, in some species it is clear that the members of that species can adapt their mating strategy to ensure a more-diverse set of children, who are thus better able to cope with a range of pathogens. The reason that they are better able to cope (as a set) is that there is simply more chance that they have some mutation (through crossover) which confers a selective advantage in being able to fight off infections against a set of pathogens. Although there are several models of influence of host contact networks on pathogen diversity, ${ }^{4-6}$ there are none (to the authors knowledge) that combine host mating strategy with effect on pathogen diversity.

In this work, I test the hypothesis that pathogens have an effect on genes governing human mating behavior, and that this has feedback into pathogenic diversity. In particular, that human mating behaviour has an effect on pathogen diversity. This synergistic behavior is typical of complex systems, where the behaviour of individuals results in nonlinear aggregate "emergent" behaviours. The feedback cycle that I am exploring is that mating strategy influences the human population genetic diversity that the viruses have to target to survive, and this resulting viral population then imposes selection pressure on the human population, including genes for mating strategy. In previous work I studied human sexual contact networks with a spread of viruses, and a spread of viruses and mutable ideas in separate work. This work combines those two topics to explore this particular hypothesis.

I considered that humans have a gene "for" infidelity. When I (and others, like Dawkins, ${ }^{7}$ talk of a gene being "for" something, I am saying, all other things (including other genes) being equal, that this gene influences behaviour through different types (or chemical concentration) of a protein. This is a gene for some human behavior, whether it be mating strategy or homosexuality, is only true in a statistical sense. ${ }^{8}$ In my model I have the behavior set in a deterministic sense (which would assume a very high probability that a gene for infidelity would have an effect on mating strategy).

It is also important to remember that along with genes, cultural and family background also play a large role in determining behaviour. The culture also sets up societal systems for taking care. Women face a number

Send correspondence to Matthew Berryman

E-mail: matthew@berrymanconsulting.com, Telephone: +61 413458594 
of tradeoffs in selecting mates, both for the long term and short term ${ }^{9,10}$ and these are highly dependent on culture, although it should be noted that some of these are also faced by females across the animal kingdom. This assumes a society in which women are the predominant child raisers. If she can stay in a monogamous relationship, or "get away" with cheating and still have a husband around, then this is "better" than if she doesn't have a husband around. On the other hand, a male benefits his genes more and more, the more he is unfaithful, since the women will be raising his children. So it is not surprising that a mixed strategy (which doesn't have to be used exclusively for a whole lifetime, although I simplified my simulation by doing that) typically arises in which there are some (women) who remain in semi-monogamous relationships, but there is a population of (mostly) men who cheat a lot. This whole model ignores emotions, but then in evolutionary terms, the emotions don't matter much here, since they mainly occur after children have already been raised. Thus we expect nature to not really care either way if people get hurt.

In evolutionary game theory terms we then find that good solutions spread through a population: even if they confer a slight advantage, then over time they will spread. In the following section I detail the particular method that I use to study the spread of genes in co-evolving human and viral populations. I attempt to answer the following questions about this co-evolution:

- Do pathogens affect mating strategy in human populations?

- Does the pattern of crossover alter human response to viral populations? Crossover simply refers to the pattern of selecting parts of one chromosome to combine with another.

- Do the rates of mutation effect the co-evolution?

- Does the number of viruses effect the co-evolution?

- Does the step ratio of virus generations to human generations effect the co-evolution?

\section{METHODS}

In the following subsections I discuss the way in which I represent both humans and viruses in my model, the algorithm and parameters for co-evolution that I use, and briefly mention tools used in analysing the data in preparation for graphing the results.

\subsection{Representation}

To represent chromosomes, in order to model both the types of behavior that humans use and the response of different human phenotypes to different viral strains, I treat the chromosomes as bit vectors. Both human chromosomes and viral chromosomes were 7 bits long. Humans also have a gender, which is represented by a separate bit. A score on the lethality of each virus going through the human population could thus be given as $7-h_{d}(\mathbf{x}, \mathbf{y})$ where $\mathbf{x}$ and $\mathbf{y}$ are the bit vectors for humans and viruses respectively, and $h_{d}$ is the Hamming distance between the two (argument) strings. Thus this score is determined by the number of bits the two chromosomes have in common. This score also works in reverse to determine the human fitness. Since gender is excluded from the human chromosome representation, I am thus not considering viruses with gender-specific lethality like some strains of the human papilloma virus.

The first two bits of the human chromosome are used to determine the phenotype of mating behavior of the human as follows in Table 1. The genotype, or bit pattern of those two bits uses a zero to represent the non-cheating form of the gene, and 1 the mutant form. The monogamous type is fairly clear: they haven't got any copies of the gene for cheating, so they remain totally faithful to their partner (even if their partner cheats on them). People who are heterozygotes, that is, they have only one copy of the non-cheating form, thus cheat around only some of the time. The plays around phenotype is thus when a person remains in a long-term relationship, but has a "bit on the side". If a person has two copies of the gene, then they have the serial cheater phenotype, and never have a long-term relationship, just a series of flings. A child must have a mother who is in a long-term relationship, in order that the child has some source of resources. I am thus modeling a society like my own in which women are normally the primary care-givers, and men are often absolved of their 
Table 1. This table shows the mapping between genotype (bits 0 and 1 of the 7 bit chromosome for humans) and the phenotype. A zero denotes a copy of the monogamous/non-cheating form of the gene, and a one denotes the cheating form of the gene. Heterozygotes (those with both forms) hold down a long-term relationship with some cheating, thus a mix of the other two extreme strategies.

\begin{tabular}{|c|l|}
\hline bit pattern (genotype) & phenotype \\
\hline$(0,0)$ & monogamous \\
$(0,1)$ or $(0,1)$ & plays around \\
$(1,0)$ & serial cheater \\
\hline
\end{tabular}

responsibilities. Symmetry breaking is ubiquitous in dividing the responsibility between parents, but there is no a priori reason that it must be the female that gets the primary care role. In the following subsection I discuss what all of these phenotypes mean in terms of the children that are produced and supported, and thus can co-evolve with the viruses.

\subsection{Algorithm}

The main loop of the program, which ones run generation of human evolution, is as follows. It is similar to standard evolutionary algorithms. Note that the evolution of viruses occurs at a much faster rate than the human rate, it occurs at a fixed ratio, as a sub-loop of the main program loop. I have fixed population sizes of $n_{h}$ for humans and $n_{v}$ for viruses, and these are initialized at random, and at each step, when selection occurs, I keep only the top $n_{h}$ humans and $n_{v}$ viruses at each generation of humans.

1. Partner up females with males, after randomizing the lists, up to the maximum allowed for each type of person (obviously one person if the phenotype is monogamous, for the serial cheater and plays around phenotypes the maximum is three - I assume that more relationships than this are hard to achieve (certainly for women, and also for non-"James Bond"-men) and didn't choose to explore this parameter.

2. Children are created from each partnership of a female who doesn't have the serial cheater phenotype with a male. The gender of the child is assigned at random with equal probability of male or female. The chromosome of the child is formed by either by selecting each bit at random from the corresponding position in the two parent chromosomes, or by choosing a point at random at which crossover between the two parent chromosomes occur. Only one style of chromosome generation is used per simulation run to be consistent. The default is the former, that is, selecting each bit at random from the corresponding position in the two parent chromosomes.

3. Each bit of every child chromosome is mutated (independently) with probability $p_{h}$. A mutation is a bit flip, ie $x_{k} \leftarrow \operatorname{not} x_{k}$ where $x_{k}$ is the $k^{\text {th }}$ bit of a chromosome.

4. Over $s_{v s}$ iterations, each bit of every viral chromosome is mutated (independently) with probability $p_{v}$. Note that $s_{v h}$ can be thought of (and is named later in the paper) as the step ratio of virus reproductive cycles per human reproductive cycles (generations).

5. Each child is assigned a score

$$
c_{i}=7-h_{d}\left(\mathbf{x}_{i}, \mathbf{y}_{j}\right) .
$$

where $c_{i}$ is the score for child $c_{i}, \mathbf{x}_{i}$ is their chromosome, the $\mathbf{y}_{j}$ are the viral chromosomes, and $h_{d}(\mathbf{a}, \mathbf{b})$ is the Hamming distance between two strings, or the number of places where the two strings differ. Thus when we take this distance away from the chromosome length (7) it gives us the similarity score between two strings (the number of places they have in common).

6. Similarly, each virus is assigned a score

$$
v_{j}=7-h_{d}\left(\mathbf{x}_{i}, \mathbf{y}_{j}\right)
$$


Table 2. Table of parameters used, including the default parameters used across all runs, with only one of the parameters changed in each run. Note that the number of viruses and number of humans is simply a count of all the viruses and humans in each generation. There may be identical viruses and identical humans, at random this would occur with probability $1 /\left(2^{7}\right)$ but the process of co-evolution means that this becomes higher as the humans and viruses co-evolve together.

\begin{tabular}{|c|c|c|c|}
\hline & \multicolumn{3}{|c|}{ parameter values } \\
parameter & min & default & $\max$ \\
\hline human mutation rate $\left(p_{h}\right)$ & 0.001 & 0.01 & 0.1 \\
\hline viral mutation rate $\left(p_{v}\right)$ & 0.001 & 0.01 & 0.1 \\
\hline number of viruses $\left(n_{v}\right)$ & 30 & 50 & 100 \\
\hline step ratio $s_{v h}$ & 10 & 50 & 100 \\
\hline
\end{tabular}

7. The viruses are ranked from highest score (most lethal) to lowest, and then multiply according to the formula

$$
m=4\left\lfloor\frac{\left(n_{v}-\text { rank of virus }\right)}{n_{v}}\right\rfloor,
$$

where $n_{v}$ is the maximum number of viruses allowed. This then produces $>n_{v}$ new viruses, which are then selected (in both a computer science and evolutionary sense) by picking the top $n_{v}$ of them by score. This formula was the simplest formula I could think of, and the factor of 4 was chosen by experimentation using the default set of parameters and seeing which produced the most interesting results - obviously if it is too low, there is not an effective selection (in the extreme case of 1 , then all viruses are kept), and in the case that it is too high then viral diversity is very rapidly lost.

8. The children, of which $>>n_{h}$ are produced by the above steps of the algorithm, are then ranked from lowest score (best survival) to highest and then selected by picking the top $n_{h}$.

Table 2 shows the parameters used, which in addition to altering the algorithm in the choice of crossover for children and the selection of children by virus strains, attempt to answer the following questions:

- Do pathogens affect mating strategy in human populations?

- Does the pattern of crossover alter human response to viral populations?

- Do the rates of mutation effect the co-evolution?

- Does the number of viruses effect the co-evolution?

- Does the step ratio of virus generations to human generations effect the co-evolution?

\subsection{Analysis}

In addition to doing a simple population count of humans with the three phenotypes, and viruses with a particular ID (the decimal representation of their binary string), I also use a statistic called the ShannonWeaver diversity index, ${ }^{11,12}$ which is the Shannon Entropy ${ }^{13}$ but has a denominator (and thus a logarithm base) equal to the logarithm of the total number of different possible strains $\left(2^{7}\right.$ for my viruses, and $2^{7}$ for my humans, ignoring gender differences), which gives a number between 0 (least diverse) and 1 (most diverse) of the genetic diversity of the population:

$$
D=\frac{\sum_{i=1}^{N_{s}} p_{i} \log \left(p_{i}\right)}{\log \left(N_{s}\right)},
$$

where $p_{i}$ is the frequency of a human or viral strain in the total population 


\section{RESULTS}

In the following subsections, I have explored the effect of varying both parts of the algorithm (specifically, turning on and off the selection of children by viruses, and the way in which crossover occurs in human chromosomes), and also the parameters as mentioned in Table 2.

\subsection{Default parameters and algorithm}

For the algorithm as described previously, and for the default set of parameters, the results as shown in Figures 1 and 2. The human mating strategy of cheating some of the time (playing around) dominates, as it is the most evolutionary stable, since if women cheat all the time then children cannot survive, and if no one cheats then not as many children are raised. The viruses are able to quickly track the evolving human population, the lack of diversity indicated does not mean an unchanging virus population (results for this not shown) but rather that they converge on a solution that is effective across a not entirely heterogeneous human population.

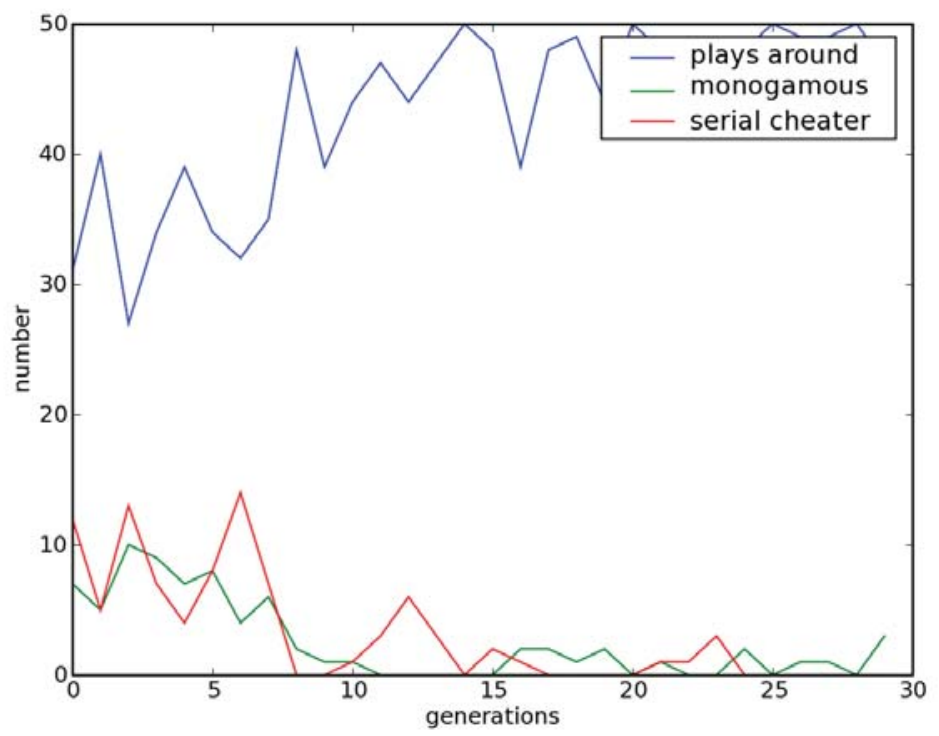

Figure 1. Human mating strategies, for the default parameters and algorithm.

\subsection{Do pathogens affect mating strategy in human populations?}

Here I switched off the selection of humans by viruses, to show the effect, as can be seen in Figures 3 and 4 . The only selection pressure on humans is the fixed population size, which means that the mating strategy (or phenotype) of playing around still dominates as described in the previous subsection. Comparing with the graphs in Figures 1 and 2, we can see that indeed that co-evolution is occurring-when we allow for co-evolution the human population diversity is reduced by the viral population, however the mating strategy appears largely unaffected by the viral population, because the viral population is mutating rather quickly, and also the mating strategy is largely dominated by the effect mentioned above where the plays around strategy is evolutionary stable even without viruses.

\subsection{Does the pattern of crossover alter human response to viral populations?}

The pattern of crossover appears to have no discernible effect on either population. 


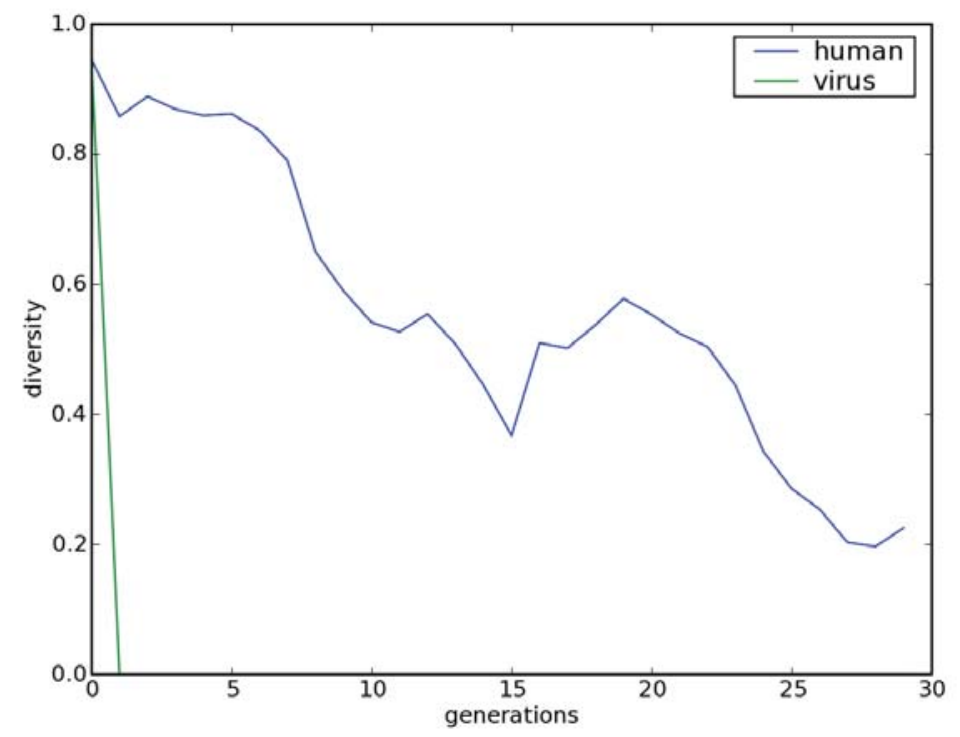

Figure 2. Genetic diversity of the human and virus populations, for the default parameters and algorithm.

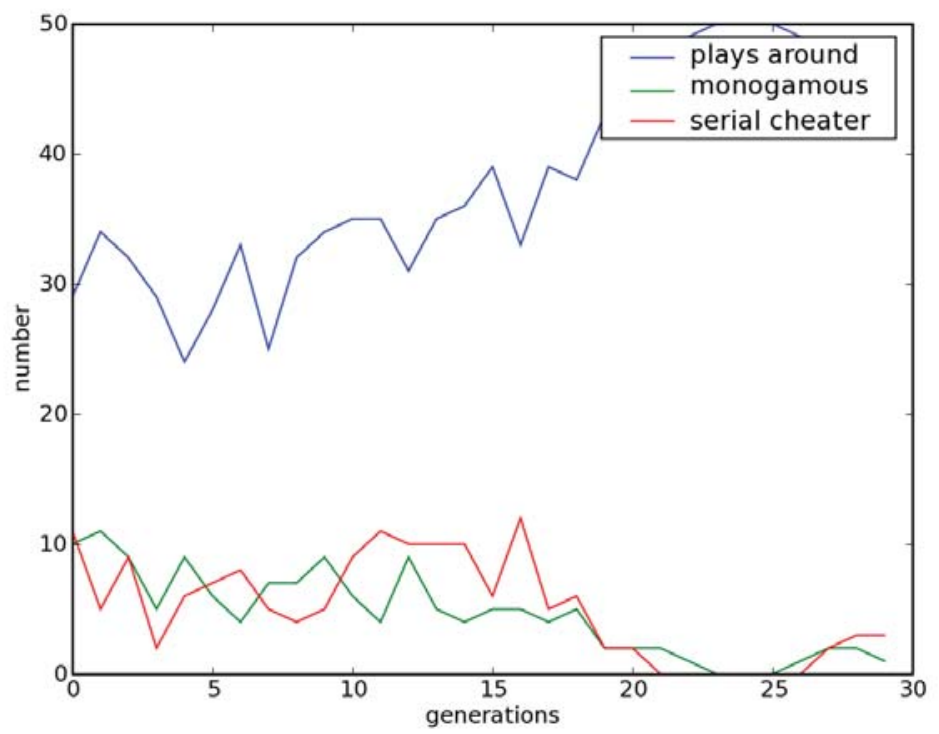

Figure 3. Populations of humans following the three different mating strategies (phenotypes), with no feedback from the virus population on the human population.

\subsection{Do the rates of mutation affect the co-evolution?}

The results of changing the rates of human mutation and virus mutation can be seen in Figures 7 and 8 respectively. Increasing either the human rate of mutation or the virus rate of mutation causes the human population to die off. Obviously if the rate of viral mutation increases then the viruses can more quickly evolve to the changing human population and are therefore end up with a higher lethality. I am unable to clearly explain why the increase in human mutation rate kills off the population. However, perhaps, as with the 


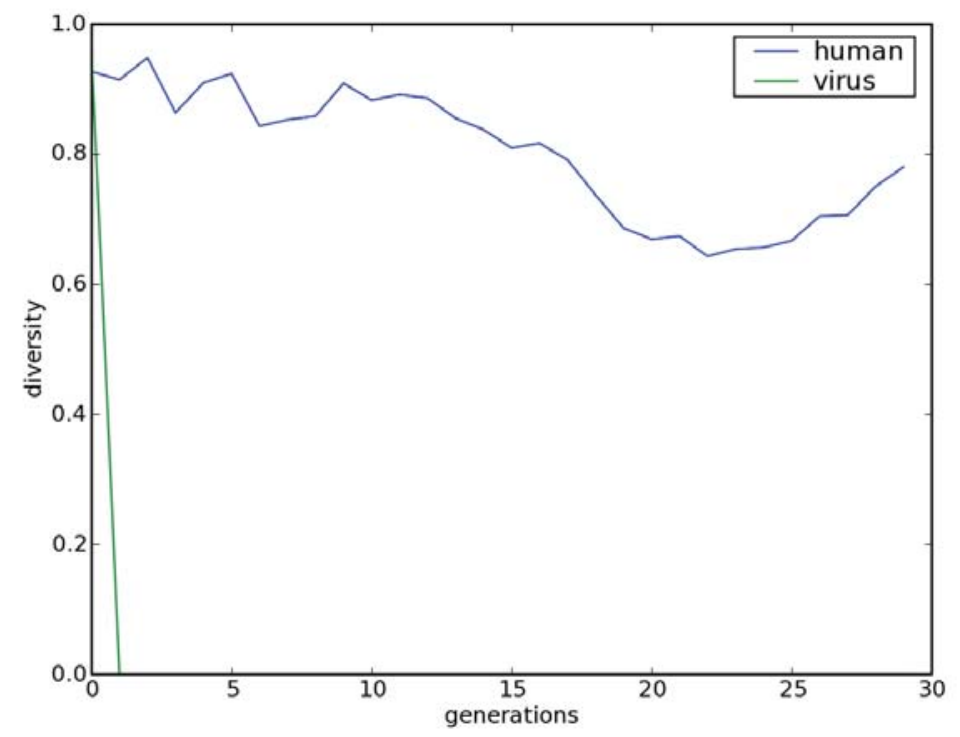

Figure 4. Genetic diversity of the human and virus populations, with no feedback from the virus population on the human population, but still with feedback from the virus population on the human population.

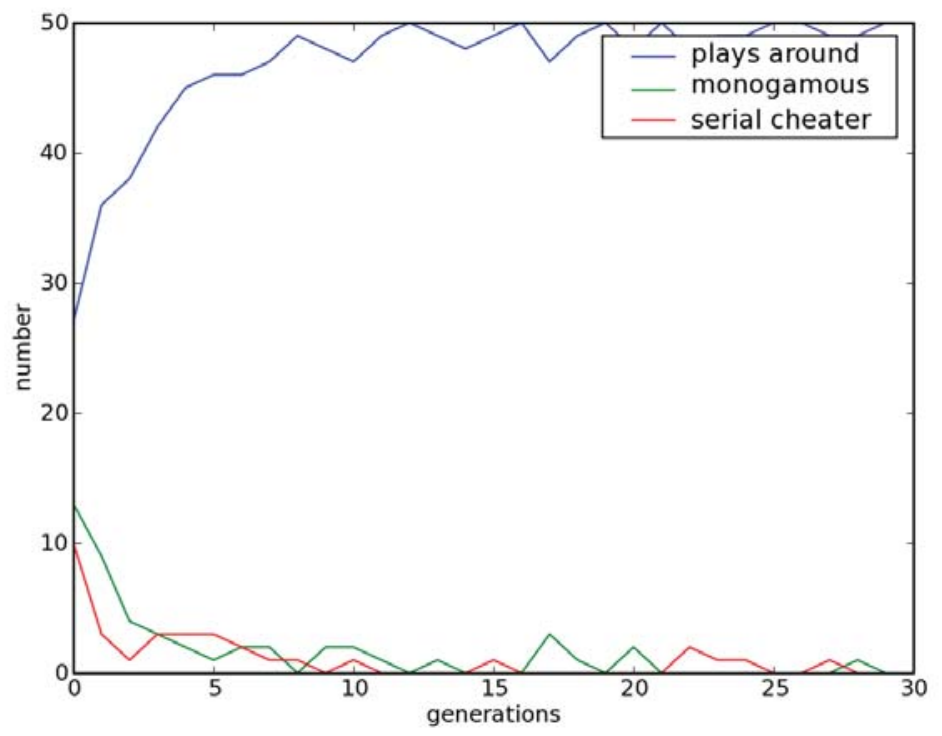

Figure 5. Populations of humans following the three different mating strategies (phenotypes), with the crossover strategy being that where a single point of crossover occurs, instead of randomly crossing over at each bit of the chromosome.

decrease in mutation rate, it affects the rate of convergence on different mating strategies, and when increased leads to some populations with too many monogamous relationships with an overall reduction in the number of children produced in each time step. 


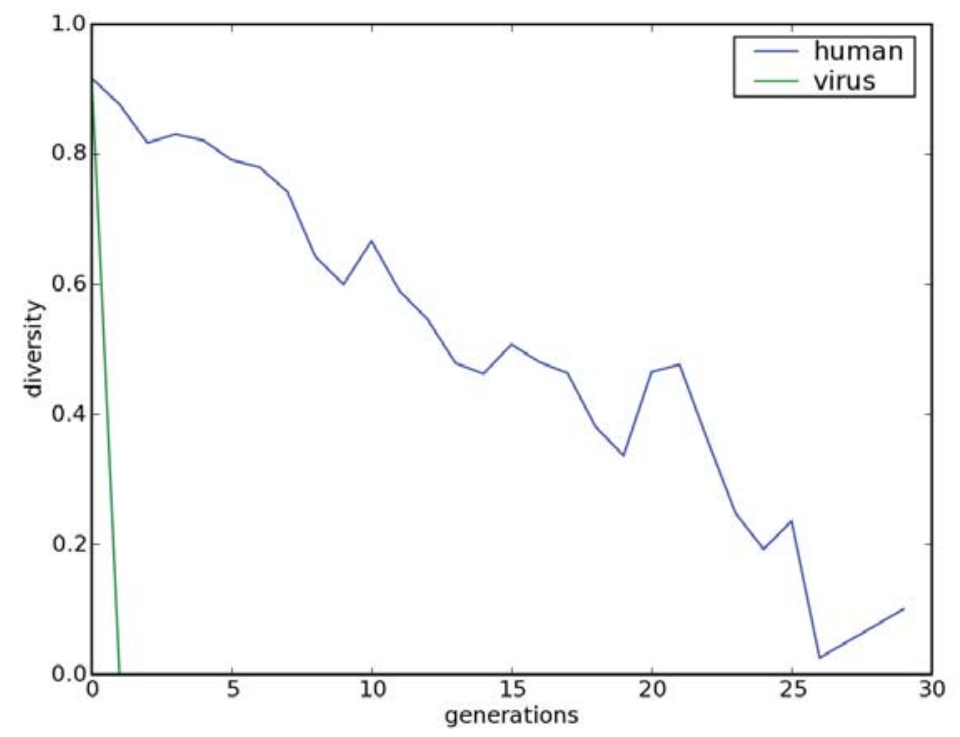

Figure 6. Genetic diversity of the human and virus populations, with the crossover strategy being that where a single point of crossover occurs, instead of randomly crossing over at each bit of the chromosome.

\subsection{Does the number of viruses affect the co-evolution?}

The number of viruses has no effect on the choice of mating strategy for reasons mentioned previously. It has a slight effect on the diversity of the human population, as a larger number of viruses allows for greater diversity of viruses, and this reduces the number of successful human chromosomes.

\subsection{Does the step ratio of virus generations to human generations affect the co-evolution?}

The step ratio of virus generations to human generations has a dramatic effect on the human population, as can be seen in Figure 10. If the viruses are allowed to evolve at a much faster rate than the human population, the human population cannot sustain enough genetic diversity to cope and everyone dies.

\section{CONCLUSIONS}

In response to each of the questions individually, I can state that:

- Pathogens have no discernible effect on human mating strategy for my default choice of parameters.

- The pattern of crossover in humans has no discernible effect on either human or pathogen populations.

- The rate of viral mutation has a predictable effect on co-evolution, in that the viruses can quickly adapt (and thus kill off) an evolving human population. The rate of human mutation effects the rate at which mating strategies propagate through a population, and if it is too high then there are too many random fluctuations of parts of the human population to the monogamous strategy and thus too few children produced to survive the presence of evolving viruses.

- More viruses means that few human genomes can survive, and genetic diversity of humans is reduced.

- The speed at which viruses evolve, either by an increased number of generations or an increased mutation rate affects the human population size, but has no discernible effect on human mating strategy. 


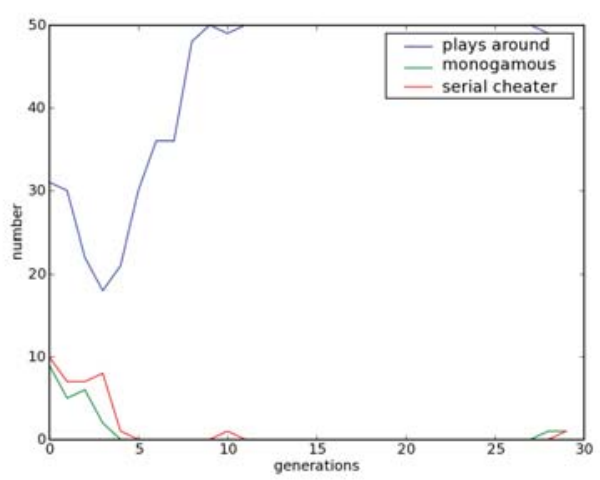

(a) Human mating strategy for $p_{h}=0.01$

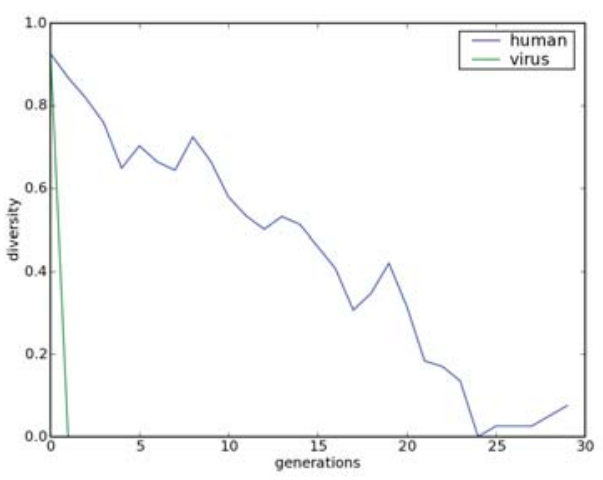

(c) Diversity for $p_{h}=0.01$

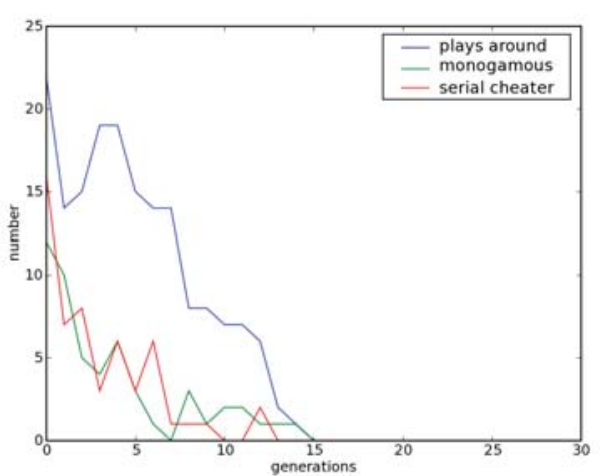

(b) Human mating strategy for $p_{h}=0.1$

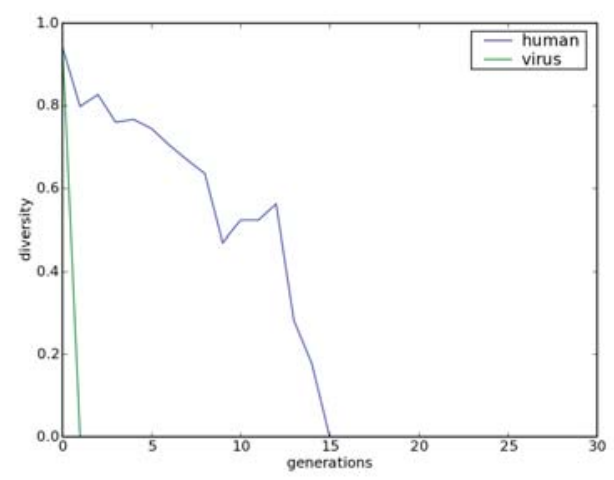

(d) Diversity for $p_{h}=0.1$

Figure 7. The effect of varying $p_{h}$, the rate of human mutation, on the human mating strategy and genetic diversity of the populations.

In summary, the co-evolution of viruses and humans is a complex system, with nonlinear and sometimes unpredictable effects. The co-evolution of viruses and humans does have an effect on human mating strategies if there is relatively high mutation rate in humans.

\section{ACKNOWLEDGMENTS}

Useful discussions with Tamath Rainsford, Axel Bender, and Alex Ryan are greatly appreciated.

\section{REFERENCES}

1. M. A. Levi and L. A. Real, "The role of resources and pathogens in mediating the mating system of Kalmia latifolia," Ecology 79(5), pp. 1602-1609, 1998.

2. N. Charbonnel, R. Rasatavonjizay, E. Sellin, P. Brémond, and P. Jarne, "The influence of genetic factors and population dynamics on the mating system of the hermaphroditic freshwater snail Biomphalaria pfeifferi," Oikos 108(2), pp. 283-296, 2005.

3. J. A. Steets and T.-L. Ashman, "Herbivory alters the expression of a mixed-mating system," American Journal of Botany 91(7), pp. 1046-1051, 2004.

4. J. M. Read and M. J. Keeling, "Disease evolution on networks: the role of contact structure.," Proc. Royal Society B: Biological Sciences 270(1516), pp. 699-708, 2003.

5. M. M. Telo da Gama and A. Nunes, "Epidemics in small world networks," Eur. Phys. J. B 50, pp. 205-208, 2006. 


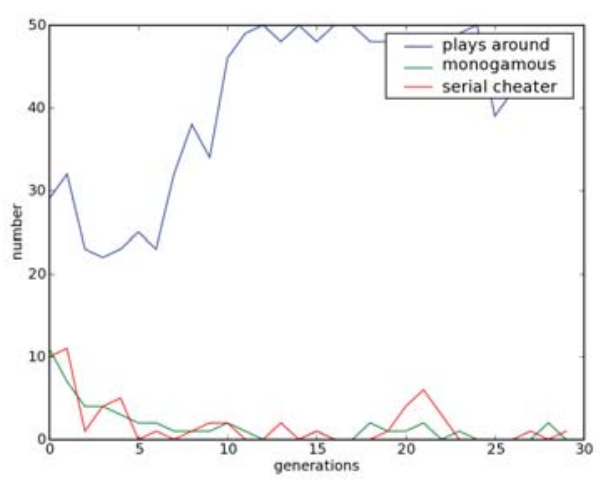

(a) Human mating strategy for $p_{v}=0.01$

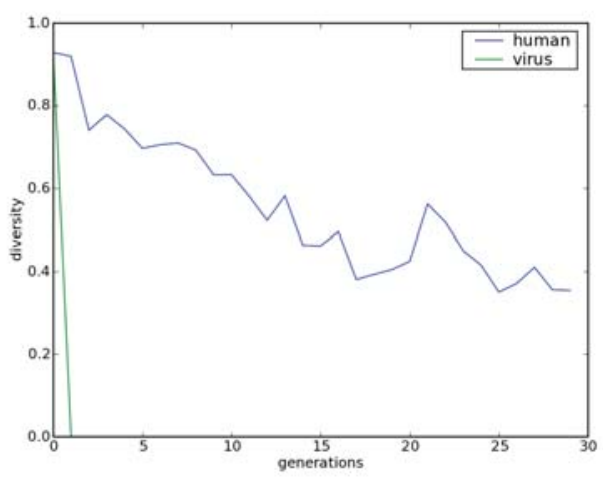

(c) Diversity for $p_{v}=0.01$

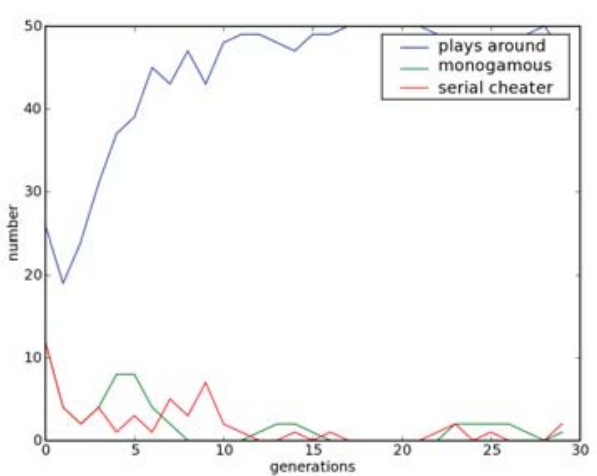

(b) Human mating strategy for $p_{v}=0.1$

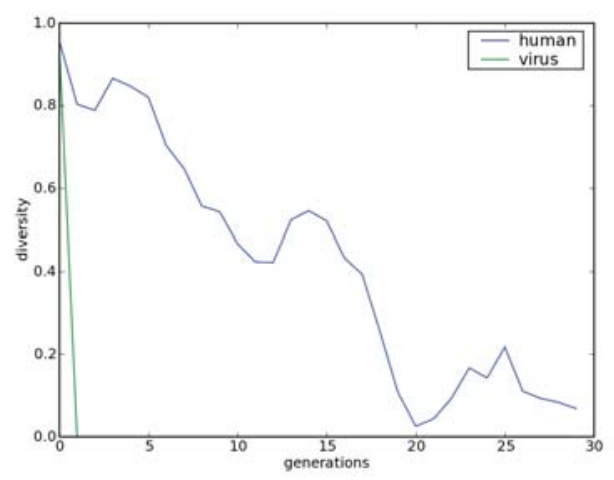

(d) Diversity for $p_{v}=0.1$

Figure 8. The effect of varying $p_{v}$, the rate of viral mutation, on the human mating strategy and genetic diversity of the populations.

6. K. J. O'Keefe and J. Antonovics, "Playing by different rules: The evolution of virulence in sterilizing pathogens," The American Naturalist 159, pp. 597-605, 2002.

7. R. Dawkins, The Selfish Gene, Oxford University Press, 1989.

8. R. Dawkins, A Devil's Chaplain, ch. Genes Aren't Us, pp. 104-106. Weidenfeld \& Nicolson, London, UK, 2003.

9. J. E. Scheib, "Context-specific mate choice criteria: Women's trade-offs in the contexts of long-term and extra-pair mateships," Personal Relationships 8, pp. 371-389, 2001.

10. S. W. Gangestad and J. A. Simpson, "The evolution of human mating: trade-offs and strategic pluralism," Behavioral and Brain Sciences 23(4), pp. 573-644, 2000.

11. C. E. Shannon and W. Weaver, The mathematical theory of communication, Univ. of Illinois Press, Urbana, IL, USA, 19949.

12. C. O. Buckee, K. Koelle, M. J. Mustard, and S. Gupta, "The effects of host contact network structure on pathogen diversity and strain structure," Proc. Natl. Acad. Sci. U.S.A. 101, pp. 10839-10844, July 2004.

13. S. Kullback, Information Theory and Statistics, Dover Publications, 1968. 


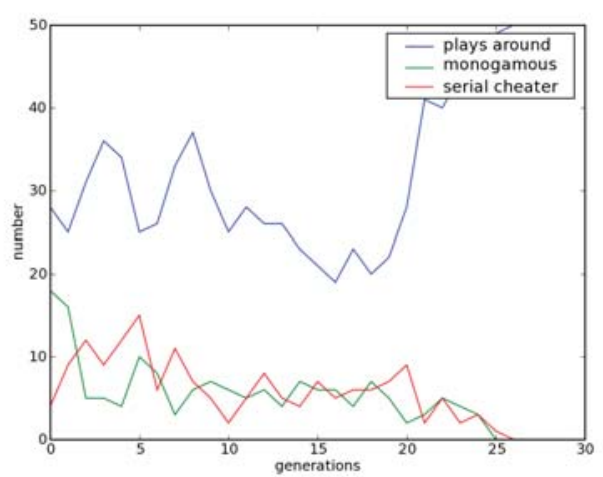

(a) Human mating strategy for $n_{v}=10$

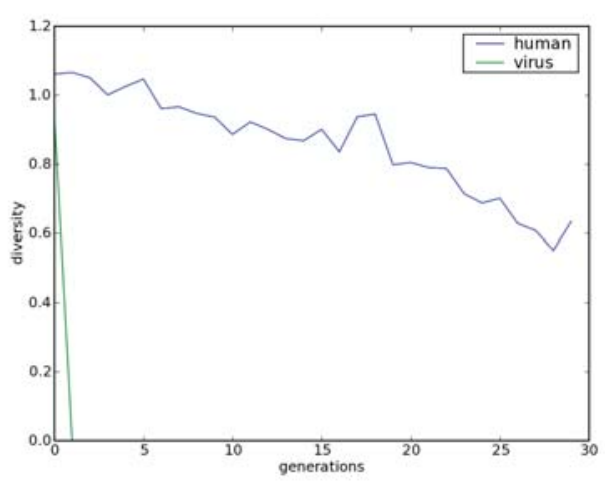

(c) Diversity for $n_{v}=10$

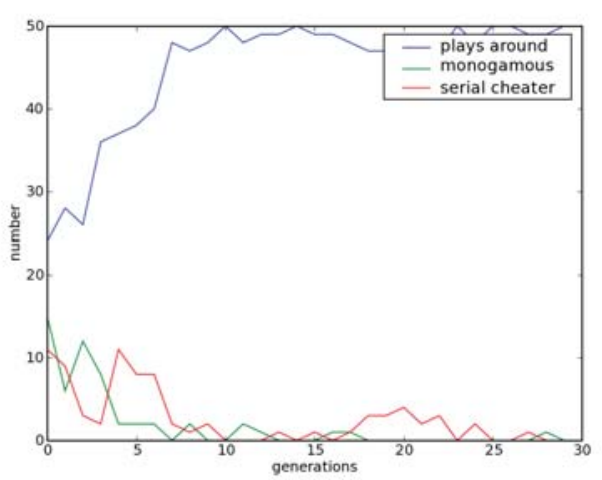

(b) Human mating strategy for $n_{v}=100$

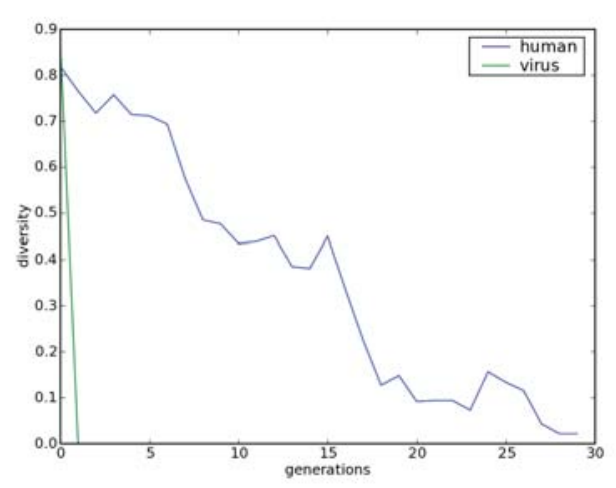

(d) Diversity for $n_{v}=100$

Figure 9. The effect of varying $n_{v}$, the number of viruses, on the human mating strategy and genetic diversity of the populations. 


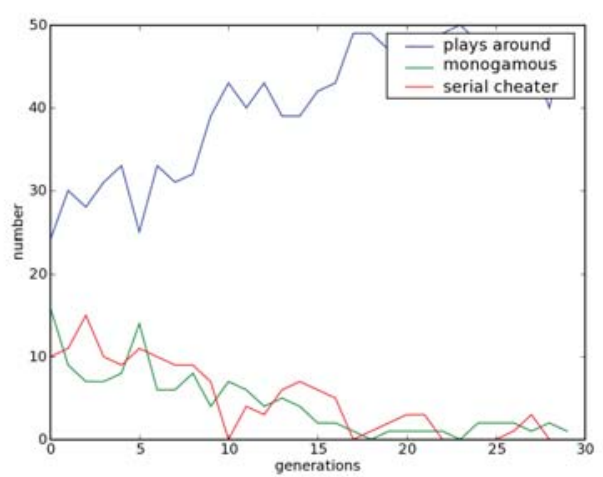

(a) Human mating strategy for $s_{v h}=10$

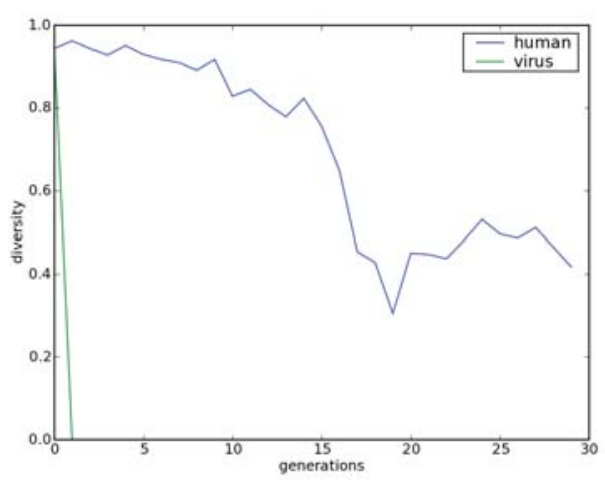

(c) Diversity for $s_{v h}=10$

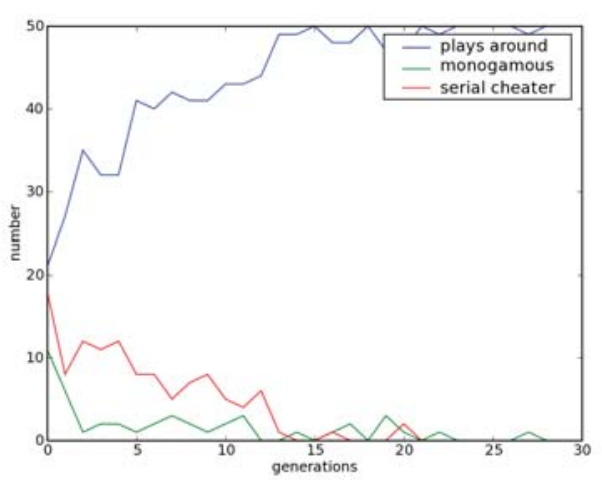

(b) Human mating strategy for $s_{v h}=100$

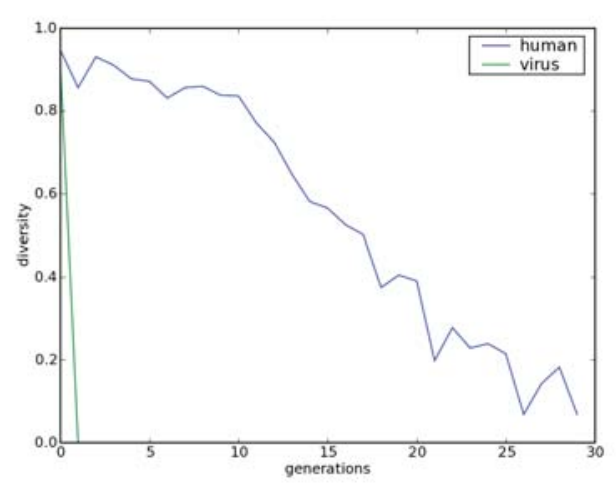

(d) Diversity for $s_{v h}=100$

Figure 10. The effect of varying $s_{v h}$, the number of generations of viruses per human generation, on the human mating strategy and genetic diversity of the populations. 